\section{AACR makes public 19,000 cancer genomes}

On 5 January, the American Association for Cancer Research (AACR) made the first public release of 19,000 de-identified genomic records obtained through its project GENIE (Genomics Evidence Neoplasia Information Exchange). The data set contains genomic data collected from cancer patients treated at eight institutions in the US, Canada, the Netherlands and France. It is among the largest fully public cancer genomic data sets released to date, the AACR said in a press release. The findings were generated from patients whose tumors were sequenced as part of their routine care. The information was then pooled into a single registry and linked to selected longitudinal outcomes. The registry encompasses patients with 59 major cancer types, including nearly 3,000 with lung cancer and more than 2,000 with colorectal cancer. GENIE was launched in November 2015. Its goal is to provide a database for scientists to generate research hypotheses spanning translational to clinical studies, including those that would inform new or ongoing clinical trials; validate biomarkers; reposition or repurpose drugs; add new mutations to existing drug labels; and identify new targets. Because it will be enriched with examples of late-stage disease, the data from GENIE could also provide payers with the evidence base necessary to support reimbursement for next-generation sequencebased testing. This initial data set is available from the AACR website or by download from informatics partner Sage Bionetworks. The consortium is now accepting applications for new participating centers to expand the registry, a year ahead of schedule.

\section{6 "If [Trump] has the stamina he will see two things... the American public is being taken advantage of. And secondly, we are funding the R\&D for free riders across the world. And I don't think the president- elect... is going to take too well to that." Centers for Medicare and Medicaid acting administrator Andy Slavitt at the JP Morgan Healthcare Conference in San Francisco in January comments on the premiums paid by US taxpayers, which underwrite cheaper drugs for European and Asian markets. (MarketWatch, 10 January 2017)}

\footnotetext{
"[The NCl Formulary] has got to work well, it's got to work fast, it's got to be frictionless." Dr. George Demetri, from the Dana-Farber Cancer Institute in Boston, reflects on the newly announced program, in which the $\mathrm{NCl}$ will set up a formulary of company assets, enabling scientists to test drug combinations. So far six pharma companies are making 13 drugs available. (STAT News, 11 January 2017)
}

comprehensive, including frequent proteomic and antibody sampling and 24-hour glucose monitoring, and affordably priced. "Some companies are doing one or a few of these things. No one is assembling it all," says Wang. The group is also training coaches to help users digest the information.

iCarbonX has so far accrued over $\$ 600$ million in investment. The original \$200 million came within a year of its October 2015 founding from Shenzhen-based Tencent, which produces WeChat, China's social media application with some 800 million users; Zhongyuan Union Stem Cell Bioengineering Company, a biotechnology company based in Tianjin; and the Tianfu Group, a Shanghai-based consulting group. iCarbonX is not divulging the source of the new $\$ 400$ million that it plowed into the seven alliance members. $\$ 7.5$ million went to HealthTell, but other amounts are undisclosed.

For each partner, the agreement and investment are different. For SomaLogic, the company will provide proteomics expertise to the alliance; iCarbonX will invest (an undisclosed amount) in the company and help to set up a SomaLogic joint venture in China. But some details need to be worked out. "We're still dancing," says SomaLogic founder Gold, who hopes it remains a tight alliance. "[Wang] is someone you'll follow gladly. You don't meet many people like that. I'm all in."

Jamie Heywood, co-founder and chairman of Cambridge, Massachusetts-based PatientsLikeMe, says he is "energized" by the alliance and by Wang's laissez faire approach. "Jun didn't put on any constraints. He is giving us an opportunity to go further and faster than we could anywhere else," says Heywood, whose company is an online portal where some 500,000 registered patients submit clinically useful data about their experience with drugs and disease. Wang plans to use PatientsLikeMe as a base for getting into the US and to create a PatientsLikeMe type portal in China. "The beauty of this ecosystem is that it's good for all of us," says Heywood.

The alliance will have to convince some observers of its uniqueness. Michael Snyder, director of the Stanford Center for Genomics and Personalized Medicine, sees the Chineseled project as just one of many-including Seattle-based Arivale, San Diego based Human Longevity Inc. and his own initiative, Integrated Personal Omics Profilingthat are adding other omics to genomics in an attempt to tailor medical knowledge to individuals (Nat. Biotechnol. 3, 887-889, 2015). "We already know that the genome is just a small part of the equation and that other types of data can be quite valuable," says Snyder.
Snyder admits, though, that the investment is impressive. "Its significance will depend on how many of people they follow and the quality of data they can garner," he says.

Wang plans to gather one million users within five years, a number that will give the alliance an edge, he says. The plan is to tap health check-up clinics as well as insurance companies in the region. Representatives from the two largest Chinese insurance companies, AIA Group and the China Life Insurance Company, were at the summit. China's size and culture-which already includes an emphasis on preventative care through traditional Chinese medicine-makes Wang confident of hitting his target. Critically, the alliance plans to recruit through the wildly popular social media application WeChat. "I will try everything and go with whatever works. No one will be able to collect at the same scale of data that I am doing," he says. "A million is a starting point, not an endpoint."

The numbers are crucial if one is hoping to get through the significant noise in such a weighty data set to find strong signals, says Todd Krueger, president of AOBiome, another alliance member based in Cambridge, Massachusetts. "It's the value density. The informational value from 50 patients to 5,000 patients to 500,000 patients rises nonlinearly."

Still, the alliance will probably start modestly, says Mark Natkin, managing director at Beijing-based Marbridge Consulting. Like Arivale, a systems biology wellness company founded by Lee Hood that has just launched a personalized weight loss program, iCarbonX may begin with nonmedical products. "It may succeed, but will also likely hedge its bets, leveraging the platform to offer personalized cosmetics or healthcare products that don't really have to stand up to clinical tests for efficacy." Precision skincare is one of four fields of focus for iCarbonX, along with precision nutrition, precision health and precision medicine.

Because many alliance members are already experienced in mapping omics data to disease and treatment choice, precision medicine will make an easy starting point. Rhoda $\mathrm{Au}$, a neurologist at Boston University and member of the Framingham Heart Study who is discussing potential collaborations with iCarbonX, says the alliance also seems ready to take on the newer and more challenging field of precision health, by bringing health monitoring to people's homes-including smart toilets (for analyzing urine or feces), smart mirrors (to map changes in body shape to aid health diagnosis) and smart robots (a chat tool). Upstart iCarbonX has an edge in this nontraditional field of precision health, Au notes, because as 